\title{
Análisis comparado de los sistemas de ayuda en opacs: tipología y tendencias
}

\author{
Por Purificación Moscoso y Francisco-Manuel García-Ortiz
}
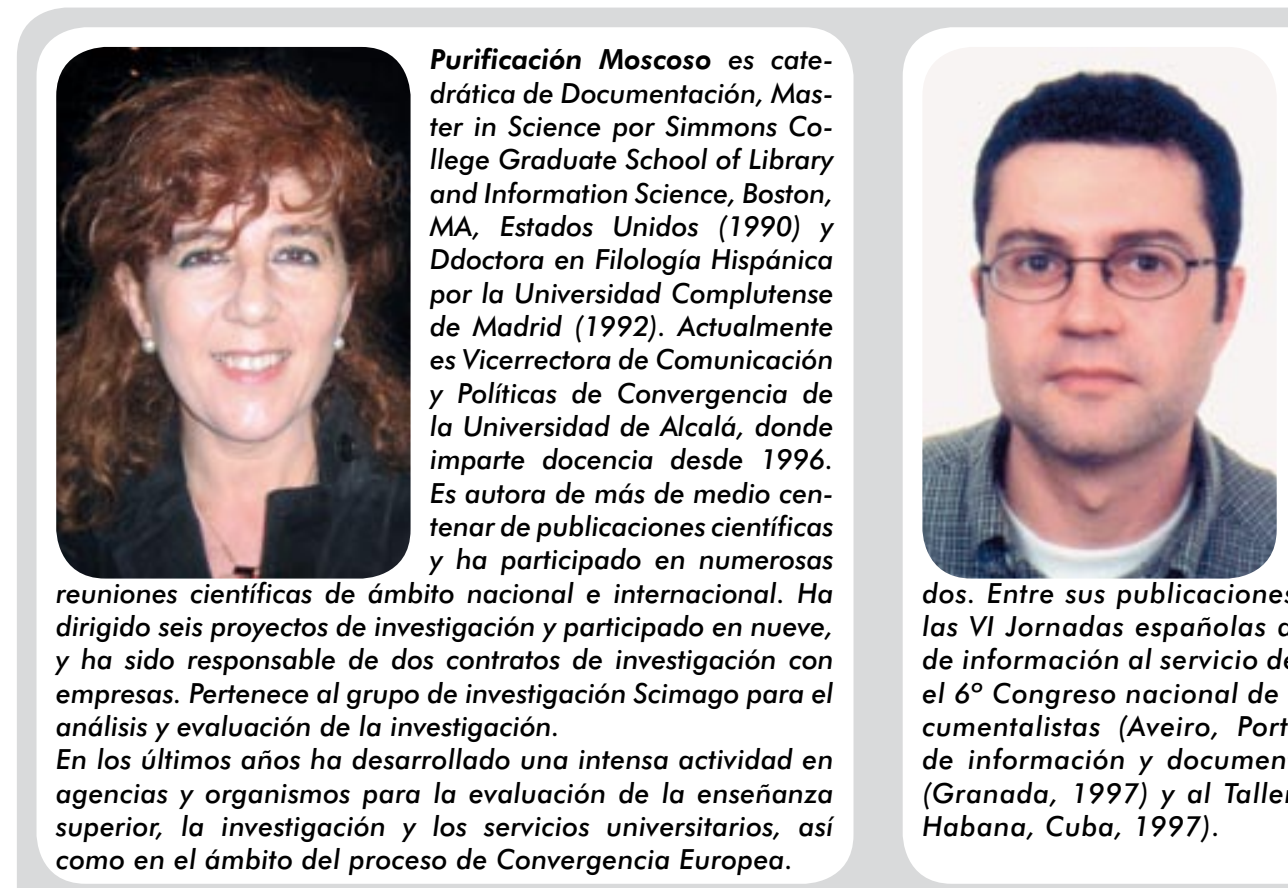

Francisco García-Ortiz es diplomado en Biblioteconomía y Documentación (1992) por la Universidad de Granada y licenciado en Documentación (1998) por la Universidad de Alcalá, donde también ha realizado el doctorado (2004). En 2005 obtuvo el Certificado profesional de bibliotecario (Washington State Library). Como bibliotecario ha trabajado, desde 1991, en el ámbito de las bibliotecas públicas, académicas y especializadas, en España y en Estados Unidos. Entre sus publicaciones destacan las contribuciones a las VI Jornadas españolas de documentación: los sistemas de información al servicio de las sociedad (Valencia, 1998), el $6^{\circ}$ Congreso nacional de bibliotecarios, arquivistas e documentalistas (Aveiro, Portugal, 1998), las VII Jornadas de información y documentación en ciencias de la salud (Granada, 1997) y al Taller internacional Qualitas'97 (La Habana, Cuba, 1997).

Resumen: Se analizan los tipos de ayuda en los OPACs de bibliotecas públicas y universitarias, de Estados Unidos y España. Se evalúan las propuestas para un sistema estándar, las herramientas de ayuda indirecta y otra información de apoyo. Se estudian las tendencias más significativas observadas en el periodo 2005-2007 y se presentan los resultados atendiendo a su distribución geográfica y tipo de biblioteca.

Palabras clave: OPAC, Catálogo en línea de acceso público, Ayuda en línea, Evaluación, Bibliotecas universitarias, Bibliotecas públicas, Estados Unidos, España

\section{Title: Comparative analysis of OPAC help systems: types and trends}

Abstract: Various types of OPAC help provided by academic and public libraries in Spain and the United States are examined. The results of this investigation are reported, by geographic distribution, library type, and catalog software. The study identifies the most relevant trends in relation to online help systems.

Keywords: OPAC, Online public access catalog, Online help, Evaluation, Academic libraries, Public libraries, United States of America, Spain

Moscoso, Purificación; García-Ortiz, Francisco-Manuel. "Análisis comparado de los sistemas de ayuda en opacs: tipología y tendencias”. En: El profesional de la información, 2007, julio-agosto, v. 14, n. 4, pp. 295-305.

DOI: 10.3145/epi.2007.jul.03

\section{Introducción}

Uno de los mayores problemas de la clásica ayuda en línea es que los usuarios no la utilizan, a pesar de que se encuentran, en más ocasiones de las aceptables, bloqueados, cuando no perdidos, ante una pantalla de búsqueda poco amigable o frente a mensajes de error confusos e imprecisos (Adams, 2001; Nielsen, 2001). Otras veces, sencillamente, desconocen las alternativas existentes para obtener los resultados deseados.
Pero la ayuda del opac (online public access catalogue) no es homogénea, ni tampoco única, y su eficiencia mejora cuanto mayor es su diversidad.

El entorno web aumenta las posibilidades de integrar herramientas que refuerzan y complementan la tradicional asistencia prestada. Hace posible, igualmente, asegurar la ubicuidad de la información; esto es, garantizar al usuario que esté donde esté podrá llegar a todas partes, porque la información puede ser accesible desde 
cualquier parte. Ha contribuido, además, a difuminar los límites y la función del opac, que al integrarse en el portal bibliotecario puede compartir tipos y puntos de acceso a la ayuda (Ebenezer, 2003; Evans, 2001).

La existencia de múltiples alternativas para el usuario en todo momento del proceso de búsqueda se ha convertido en un elemento constantemente presente en el desarrollo de las herramientas de apoyo a la búsqueda de información. Lo que no hace mucho tiempo se resolvía a golpe de ayudas contextuales, hoy se consideraría un sistema poco flexible y con escasa capacidad de adaptación a las peculiaridades y nivel de dominio siempre cambiantes de los usuarios.

\section{"La ayuda del opac no es homogénea, ni tampoco única y su eficiencia mejora cuanto mayor es la diversidad de tipos y formatos"}

La solución al problema de la ubicuidad de la información, que era fundamentalmente tecnológico, ha ampliado el concepto de ayuda, que ha pasado de centrarse en las clásicas ayudas contextuales a considerar cualquier tipo de información de apoyo. ¿Por qué no darle al usuario la distribución de accesos para que sepa que la búsqueda por materias es mucho menos usada que la de palabras clave y tome una decisión al respecto?

En este artículo se presentan los resultados del análisis de los tipos de ayuda en los opacs de bibliotecas públicas y universitarias, tomando como referencia, además de las propuestas para un sistema estándar, las consideradas herramientas de ayuda indirecta, tales como foros de opinión o servicios de referencia virtual, así como otra información de apoyo. El estudio abarca el periodo 2005-2007, lo que permite conocer las mejoras implementadas y las tendencias más significativas observadas en los dos últimos años.

\section{Material y métodos}

Para seleccionar los opacs se realizó un estudio no probabilístico, basado en un muestreo intencional y por cuotas ${ }^{1}$, aplicando los criterios de volumen de implantación (software implantado en un gran número de bibliotecas), multifuncionalidad, modernización (actualizaciones periódicas acordes con los cambios tecnológicos), geográfico (bibliotecas de Estados Unidos y España), capacidad de gestión (más de 100.000 volúmenes) e integración de funcionalidades básicas en el sistema de ayuda. Su aplicación resultó en la muestra de la tabla I.
La identificación de los tipos de ayuda se basa, fundamentalmente, en los trabajos de Adams (2001), Weber (2004) y White (1994); así como en las recomendaciones de Writers $U A^{2}$ y las aportaciones de Shneiderman (1998) y Kearsley (1988).

Los trabajos de White presentan una metodología de evaluación de opacs a partir del sistema de ayuda, entendido no como el equivalente en línea de un manual de instrucciones, sino como la interconexión de las funcionalidades de cuatro grupos de prestaciones: formación, interfaz, tipos de ayuda y mensajes de error e información.

Adams se centra en el análisis de tres formatos de ayuda (textual, gráfica e interactiva) y los diferentes tipos (para un software de edición fotográfica en el caso del trabajo referido), con la idea de demostrar que la interactiva es más efectiva. Los resultados del estudio, sin embargo, no avalan dicha hipótesis, ya que cada una presenta una cierta igualdad, y es la suma de todas ellas lo que confiere al sistema de ayuda su verdadera utilidad.

Por último, acceder a ayudas alternativas desde la interfaz, proporcionar ejemplos reales y prácticos, así como respuestas a preguntas de procedimiento y localización, entre otras, son, para Weber, condiciones necesarias para garantizar la eficacia de cualquier sistema de ayuda en línea.

Para analizar los tipos de ayuda se utilizó un método cualitativo, mediante un formulario/plantilla basado en un protocolo, estructurado en las variables objeto de estudio, que se definen como categóricas nominales (las del tipo de respuesta “sí/no”). Las ayudas examinadas se explican a continuación:

Ayuda sensible al contexto: ofrece asistencia conforme a la actividad que se esté realizando. Se ha evaluado su disponibilidad en las pantallas de búsqueda, resultados y registros individualizados.

Ayuda en formato multimedia: la ayuda puede ofrecerse o complementarse, mediante gráficos, imágenes, animación o sonidos. Especialmente útiles para usuarios con ciertas discapacidades o niños, por ejemplo.

Boletines electrónicos de novedades: al incluir una limitación temporal en la búsqueda, facilitan la recuperación de material de reciente incorporación.

Burbuja informativa: proporciona una explicación del icono, frase o término sobre el que se posa el ratón, mediante una breve descripción, incluyendo ejemplos o haciéndola interactiva, lo que resulta idóneo para mejorar las funcionalidades formativas del opac.

"Cómo puedo..." (How do I...): indica, a través de ejemplos, el modo de realizar una operación. Muy co- 


\begin{tabular}{|c|c|c|c|}
\hline Nombre de la biblioteca & Tipo de biblioteca & País & Software \\
\hline University of Oregon Libraries & Académica & EE.UU. & \multirow{15}{*}{ Innopac (Millenium) } \\
\hline University of Washington Libraries & Académica & EE.UU. & \\
\hline University of Arizona Library & Académica & EE.UU. & \\
\hline Ohio University Libraries & Académica & EE.UU. & \\
\hline American Museum of Natural History. Research Library & Académica & EE.UU. & \\
\hline Biblioteca de la Universidad Complutense de Madrid & Académica & España & \\
\hline Biblioteca de la Universidad de Granada & Académica & España & \\
\hline Biblioteca de la Universidad de Salamanca & Académica & España & \\
\hline Biblioteca de la Universidad de Sevilla & Académica & España & \\
\hline Arlington Heights Memorial Library & Pública/Regional & EE.UU. & \\
\hline Houston Public Library & Pública/Regional & EE.UU. & \\
\hline Brooklyn Public Library & Pública/Regional & EE.UU. & \\
\hline Belmont Public Library & Pública/Regional & EE.UU. & \\
\hline Santa Clara City Library & Pública/Regional & EE.UU. & \\
\hline Biblioteca Foral de Bizkaia & Pública/Regional & España & \\
\hline University of Oklahoma Libraries & Académica & EE.UU. & \multirow{14}{*}{ SirsiDynix $®($ Unicorn $®)$} \\
\hline University of New Orleans Library & Académica & EE.UU. & \\
\hline Libraries of Montana State University-Bozeman & Académica & EE.UU. & \\
\hline A.A. Lemieux Library Seattle University & Académica & EE.UU. & \\
\hline Florida Institute of Technology Evans Library & Académica & EE.UU. & \\
\hline Biblioteca de la Universidad de Alcalá & Académica & España & \\
\hline Biblioteca de la Universidad Autónoma de Madrid & Académica & España & \\
\hline Biblioteca de la Universidad Carlos III de Madrid & Académica & España & \\
\hline Biblioteca de la Universidad de Alicante & Académica & España & \\
\hline Cleveland Public Library & Pública/Regional & EE.UU. & \\
\hline Kansas Public Library & Pública/Regional & EE.UU. & \\
\hline Rocky River Public Library & Pública/Regional & EE.UU. & \\
\hline District of Columbia Public Library & Pública/Regional & EE.UU. & \\
\hline Richland Public Library & Pública/Regional & EE.UU. & \\
\hline Austin Peay State University Library & Académica & EE.UU. & \multirow{10}{*}{ SirsiDynix $®$ (Horizon) } \\
\hline Tacoma Community College Library & Académica & EE.UU. & \\
\hline Utah State University Libraries & Académica & EE.UU. & \\
\hline Buena Vista University Library & Académica & EE.UU. & \\
\hline University of Chicago Library & Académica & EE.UU. & \\
\hline Boston Public Library & Pública/Regional & EE.UU. & \\
\hline Ocean County Library & Pública/Regional & EE.UU. & \\
\hline Harris County Public Library & Pública/Regional & EE.UU. & \\
\hline Timberland Regional Library & Pública/Regional & EE.UU. & \\
\hline Linda Hall Library & Pública/Regional & EE.UU. & \\
\hline Arapahoe Community College Library & Académica & EE.UU. & \multirow{10}{*}{$\begin{array}{l}\text { CARL.Solution y } \\
\text { Library. Solution }\end{array}$} \\
\hline Parkland College Library & Académica & EE.UU. & \\
\hline Vernon College Library & Académica & EE.UU. & \\
\hline Baltimore Hebrew University Library & Académica & EE.UU. & \\
\hline John Brown University Library & Académica & EE.UU. & \\
\hline Broward County Library & Pública/Regional & EE.UU. & \\
\hline Los Angeles Public Library & Pública/Regional & EE.UU. & \\
\hline Phoenix Public Library & Pública/Regional & EE.UU. & \\
\hline Chicago Public Library & Pública/Regional & EE.UU. & \\
\hline Denver Public Library & Pública/Regional & EE.UU. & \\
\hline Brooklyn College Library & Académica & EE.UU. & \multirow{5}{*}{$\begin{array}{l}\text { Ex-libris } \\
\text { Aleph } 500\end{array}$} \\
\hline Harvard University Library & Académica & EE.UU. & \\
\hline University of Iowa Libraries & Académica & EE.UU. & \\
\hline Boston College Libraries & Académica & EE.UU. & \\
\hline Florida International College Libraries & Académica & EE.UU. & \\
\hline Biblioteca de la Universidad de Castilla-La Mancha & Académica & España & \multirow{5}{*}{$\begin{array}{l}\text { Baratz } \\
\text { Absys } 6\end{array}$} \\
\hline Biblioteca de la Universidad de Murcia & Académica & España & \\
\hline Biblioteca Central del Instituto Cervantes & Académica & España & \\
\hline Biblioteca Pública de Valladolid & Pública/Regional & España & \\
\hline Biblioteca de Andalucía & Pública/Regional & España & \\
\hline
\end{tabular}

Tabla 1. Listado de OPACs evaluados 
mún en los sitios web, sobre todo en los denominados "de servicios". Da respuesta a uno de los tipos de preguntas, en este caso de procedimiento y de las más comunes, al que todo sistema de asistencia debe atender.

Directorio electrónico: listado de correos electrónicos que permite comunicarse con el personal de la biblioteca.

Ejemplos en las pantallas de ayuda: Pueden aparecer en cualquiera de las pantallas de búsqueda y su eficacia mejora cuando se destacan, $\mathrm{y}$, en determinados casos, cuando además se utilizan formatos gráficos y/o animados.

"Encontrar": busca información dentro de la página o páginas donde se realiza la consulta. Muy habitual no sólo en los sitios web, sino también en softwares de uso común, como los procesadores de texto.

Explicación sobre la ordenación de las colecciones: ayuda a localizar la ubicación de los ejemplares (ejemplo 5).

Explicación de los sistemas documentales: ayuda a realizar las búsquedas por materias (la que genera más fallos) al proporcionar una explicación sobre los sistemas utilizados para la asignación de descriptores o encabezamientos de materia (ejemplo 1$)^{3}$.

FAQs: acrónimo inglés (frequently answered -o asked-questions) referido a las preguntas frecuentes planteadas por los usuarios con sus correspondientes respuestas. Como tipo de ayuda se localizaría, por ejemplo, junto a los casilleros de búsqueda, para dar respuesta a preguntas comunes relativas al proceso de recuperación.

Guía de ayuda personalizada: adaptación de la guía general de ayuda para grupos concretos de usuarios (niños e inmigrantes, en las bibliotecas públicas, o estudiantes de primer año en las académicas, por ejemplo).

Herramientas de participación y comunicación: empiezan a incorporarse en los opacs fruto de la convergencia de los servicios bibliotecarios con otros disponibles en Internet. Permiten la comunicación directa entre usuarios y entre éstos y bibliotecarios. Se ha evaluado la disponibilidad de foros y blogs (o bitácoras), así como de chats y videoconferencia, que permiten prestar servicios de referencia virtual (ejemplo 2) . $^{4}$

Índice general de ayuda: lista alfabética que da acceso a los contenidos de la ayuda en línea.

Índice de funciones: relación de funciones o propiedades ejecutables, incluyendo o no, una explicación o ejemplo. Son el equivalente a un "listado de instrucciones" del sistema.

Información corporativa: guías, boletines u otros documentos con información institucional, servicios, actividades, materiales e instalaciones de la biblioteca.
Información comunitaria: puesto que la biblioteca no es un ente aislado, facilitar el acceso a información de índole diversa sobre la universidad, ciudad o región puede ser de utilidad.

Listado explicativo de iconos: relación de los elementos gráficos (iconos) con su explicación correspondiente.

Mapa de localización (o "índice del sitio web”): representación gráfica o textual de un sitio web, que permite situarse dentro del contenido estructural de la página. Da respuesta a una de las preguntas frecuentes en los procesos de navegación “¿dónde estoy?”, comunes en los usuarios menos expertos y que el entorno hipertextual ha acentuado.

Mecanismos para recabar la satisfacción y opinión de los usuarios: tienen un intrínseco potencial de ayuda, pues animan (o lo contrario) a utilizar determinadas prestaciones, por ejemplo ${ }^{5}$. Sirven, además, para mejorar la asistencia del opac.

Páginas temáticas: contenidos organizados en forma de dossier sobre temas seleccionados, que sirven como vía de comunicación periódica entre la biblioteca y los intereses temáticos de grupos de usuarios.

Sistema de difusión de información por e-mail: permite informar sobre noticias, novedades y asuntos relacionados con la cuenta de usuario, así como sobre otros aspectos relativos a la institución y de interés general.

\section{Resultados generales y por tipo de biblioteca}

En 2005, más del 70\% de los opacs analizados ya incluía puntos de acceso a la ayuda que configuran la estructura tradicional de la misma: relación básica de asistencias o "puertas de entrada", instrucciones del sistema, "encontrar", burbuja informativa, ejemplos y mapa de localización, entre otros (tabla $\mathrm{II}^{6}$ ). En estos tipos de ayuda no se observaban diferencias significativas entre los catálogos universitarios y los de bibliotecas públicas.

Ahora bien, las bibliotecas no explotaban el potencial de las tecnologías, que, a tenor de los desarrollos

"La evolución de servicios en la Red, con potentes y sofisticados sistemas de asistencia, ha favorecido la implementación de un nuevo concepto de ayuda en los catálogos, más amplio y resultado de la integración de prestaciones de índole muy diversa" 


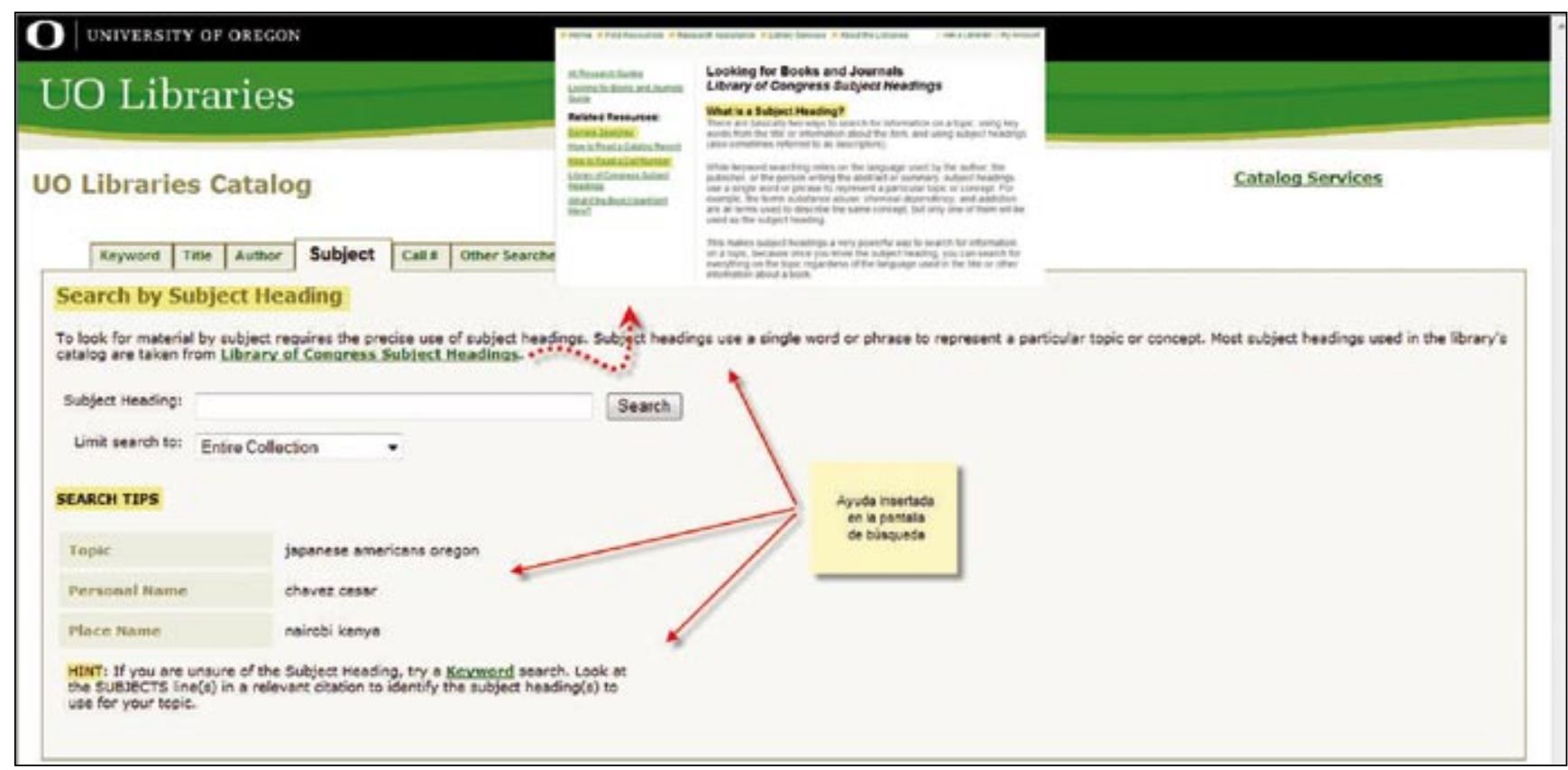

त Home त Find Resources $\pi$ Research Assistance त Library Services $\pi$ About the Libraries

:: Ask a Librarian :: My Account

All Research Guides

Looking for Books and Journals

$\underline{\text { Guide }}$

\section{Related Resources:}

Sample Searches

How to Read a Catalog Record

How to Read a Call Number

Library of Congress Subject

Headinas

What if the Book I Want Isn't Here?

\section{Looking for Books and Journals Library of Congress Subject Headings}

\section{What is a Subject Heading?}

There are basically two ways to search for information on a topic: using key words from the title or information about the item, and using subject headings (also sometimes referred to as descriptors).

\begin{abstract}
While keyword searching relies on the language used by the author, the publisher, or the person writing the abstract or summary, subject headings use a single word or phrase to represent a particular topic or concept. For example, the terms substance abuse, chemical dependency, and addiction are all terms used to describe the same concept, but only one of them will be used as the subject heading.
\end{abstract}

This makes subject headings a very powerful way to search for information on a topic, because once you know the subject heading, you can search for everything on the topic regardless of the language used in the title or other information about a book.

Ejemplo 1. Pantalla de ayuda que explica cómo buscar usando los LCSH, en el opac de la Universidad de Oregón (ampliada la ventana de la ayuda)

presentes en otros productos y servicios web, les permitía modificar los puntos de acceso, diversificar los tipos de ayuda y prestarla en formatos alternativos y complementarios al textual. Dejando a un lado las razones de índole técnica, económica o de personal, parecía existir una aceptación generalizada de que las herramientas de ayuda estándar cubrían las necesidades de los usuarios.

Ahora son muchos más los catálogos que facilitan a sus usuarios ayudas alternativas, los que integran herramientas y prestaciones complementarias a la ayuda tradicional y los que incluyen algún tipo de ayuda en formatos multimedia, gracias a herramientas de pre- sentación más ágiles y fáciles de publicar (tabla II). Se observa, además, una evolución favorable en ambos grupos de bibliotecas (gráficos 1 y 2).

El panorama ha cambiado, pero no lo suficiente para concluir que en la actualidad la asistencia que prestan los opacs converge plenamente con la proporcionada por otros softwares o servicios de la Red familiares a los usuarios.

El número de catálogos que utiliza formatos multimedia o con algún tipo de animación para facilitar la comprensión de un proceso se ha multiplicado por cinco, pero siguen sin aprovecharse sus posibilidades para mejorar la eficacia de los ejemplos o de la burbuja 


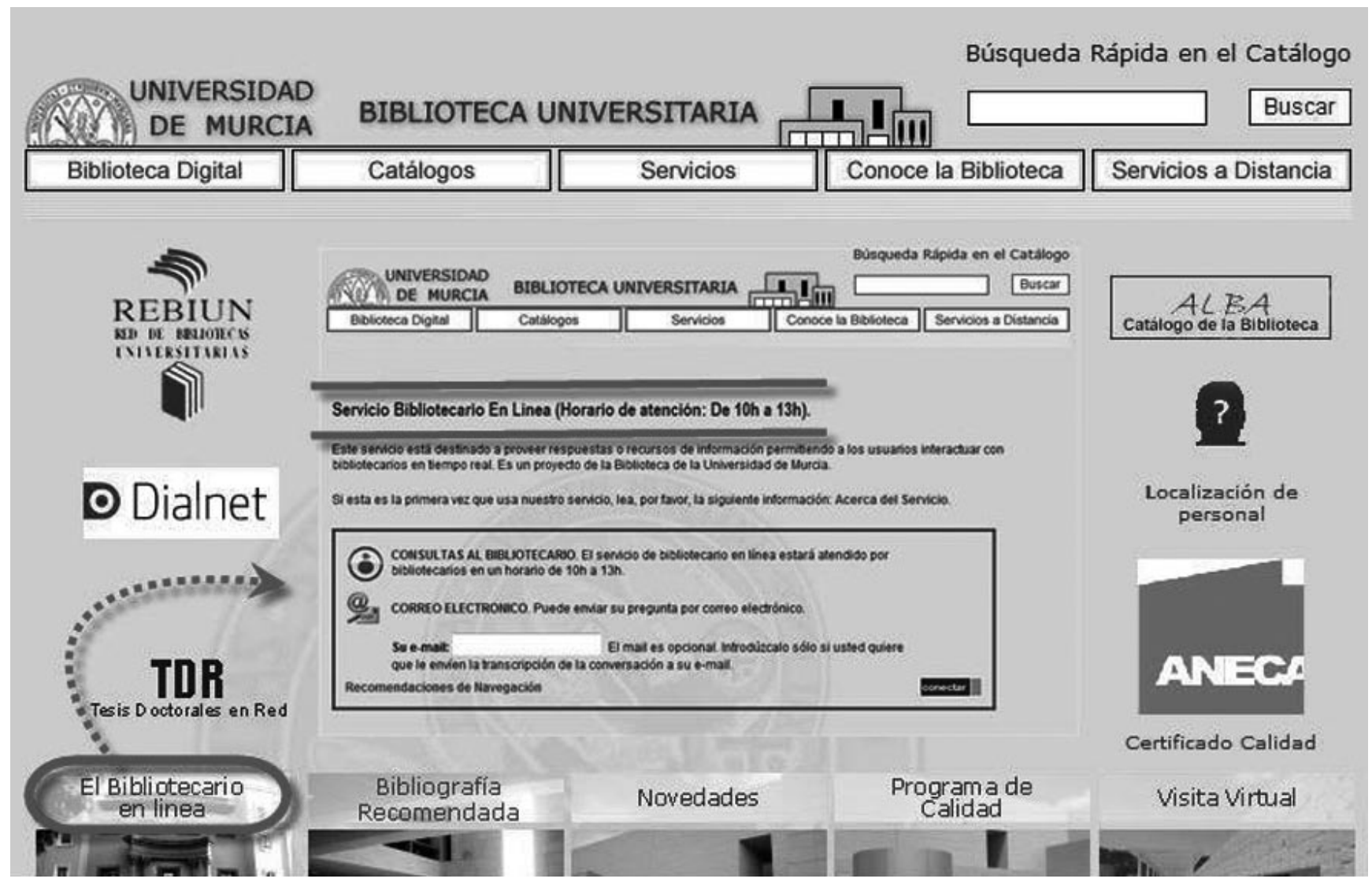

Ejemplo 2. Pantalla del servicio de referencia en línea de la biblioteca de la Universidad de Murcia

informativa, que en la mayoría se reduce a una breve explicación del término o icono correspondiente.

No llegaban a la mitad los que disponían de un índice general de ayuda o facilitaban la opción "cómo puedo...", a la que desde 2005 ya concedían mayor importancia las bibliotecas universitarias. En esta opción se observa una mejora, especialmente en las bibliotecas públicas, pero insuficiente si se considera su gran utilidad. Porque aunque esta ayuda la proporcionan también los índices de funciones, no lo hacen siempre de forma eficaz, por dos razones, fundamentalmente. La primera tiene que ver con la necesidad de que el usuario "reconozca" la función (y su nombre) sobre la que necesita ayuda. La segunda con el tipo de vocabulario empleado, en general muy técnico y ajeno al propio de un usuario cualquiera. De hecho, la confección de estos índices con un lenguaje libre de tecnicismos es una mejora necesaria en una gran mayoría de opacs.

El incremento de catálogos que han incorporado un índice general de ayuda se considera muy positivo, ya que se trata de uno de los accesos más utilizados ${ }^{7}$. Son el doble los que ahora incluyen un listado explicativo de iconos, pero todavía dos tercios de los analizados no lo facilitan. Es cierto que la tendencia a utilizar iconos "interpretativos", caracterizados, en principio, por no necesitar un texto adicional que los explique, minimiza la necesidad de este tipo de ayuda. Sin embargo, lo evidente está muy relacionado con la experiencia, el extracto social o los ámbitos geográficos y culturales. Los opacs en entorno web multiplican el abanico de sus posibles usuarios, por lo que es deseable incluir una ayuda de estas características, a fin de garantizar la comprensión de estos elementos gráficos a todos los usuarios.

\section{"Los actuales opacs disponen de sistemas de ayuda más flexibles y adaptables a las singularidades y nivel de dominio de los usuarios"}

Los resultados relativos a la implementación de servicios que permiten la comunicación directa entre usuarios y personal de la biblioteca vislumbraban una tendencia (más clara en las bibliotecas públicas) a integrar en los opacs herramientas de ayuda comunes en los servicios de la Red. Pero las mejoras observadas no pueden considerarse acordes con las posibilidades del desarrollo tecnológico ni con lo que ocurre fuera del ámbito bibliotecario, fundamentalmente en lo que se refiere a la disponibilidad de un servicio de referencia virtual, ya que casi la mitad de los estudiados carece de él.

La tendencia referida se corroboraba al analizar las FAQs, presentes en 7 y 5 de cada diez catálogos de bi- 


\begin{tabular}{|c|c|c|}
\hline \multirow{2}{*}{ Tipos de ayuda } & \multicolumn{2}{|c|}{$\begin{array}{c}\text { Resultados } \\
\text { generales (\%) }\end{array}$} \\
\hline & 2005 & 2007 \\
\hline Ayuda sensible al contexto & 46 & 58 \\
\hline Ayuda en formato multimedia & 6 & 44 \\
\hline Boletines electrónicos & 83 & 93 \\
\hline Burbuja informativa & 80 & 100 \\
\hline Cómo puedo... & 48 & 61 \\
\hline Directorio electrónico & \multicolumn{2}{|c|}{86} \\
\hline Ejemplos en pantallas de ayuda & \multicolumn{2}{|c|}{98} \\
\hline Encontrar & 80 & 90 \\
\hline $\begin{array}{l}\text { Explicación de la ordenación de las } \\
\text { colecciones }\end{array}$ & 64 & 68 \\
\hline $\begin{array}{l}\text { Explicación de los sistemas } \\
\text { documentales }\end{array}$ & 29 & 42 \\
\hline FAQ's & 59 & 73 \\
\hline Foros de participación & 7 & 34 \\
\hline Guías de ayuda personalizada & 68 & 78 \\
\hline Índice general de la ayuda & 40 & 71 \\
\hline Listado de instrucciones & \multicolumn{2}{|c|}{98} \\
\hline Listado explicativo de iconos & 15 & 32 \\
\hline Mapa de localización & 70 & 75 \\
\hline Páginas temáticas & 76 & 78 \\
\hline Referencia virtual & 37 & 51 \\
\hline Sistema difusión información e-mail & 60 & 95 \\
\hline Sugerencias y reclamaciones & 95 & 100 \\
\hline \multicolumn{3}{|c|}{ INFORMACIÓN CORPORATIVA } \\
\hline Agenda de actividades & 73 & 95 \\
\hline Fotos, imagenes & 64 & 70 \\
\hline Encuestas de opinión & 12 & 15 \\
\hline Estadísticas & 46 & 71 \\
\hline Guía de servicios & 98 & 98 \\
\hline Guía del personal & \multicolumn{2}{|c|}{93} \\
\hline Guía de la organización & 89 & 98 \\
\hline Instalaciones & 81 & 83 \\
\hline Memoria & 39 & 47 \\
\hline Normas de servicios básicos & 98 & 100 \\
\hline Noticias propias & 91 & 95 \\
\hline Reglamentos & 63 & 76 \\
\hline Revistas propias & 24 & 47 \\
\hline \multicolumn{3}{|c|}{ INFORMACIÓN COMUNITARIA } \\
\hline Agenda cultural & 32 & 41 \\
\hline Información & 51 & 93 \\
\hline Noticias externas & 24 & 73 \\
\hline
\end{tabular}

Tabla II. Resultados comparados 2005/2007

bliotecas públicas y académicas, respectivamente. $\mathrm{Su}$ progresiva implementación ha resultado en que de los analizados ya cuenta con este servicio un $73 \%$. En lo que respecta a los foros la mejora también es significativa, pero no lo suficiente si se tiene en cuenta que éstos posibilitan una asistencia mutua que, en ocasiones, puede ser más útil que la que presta el personal bibliotecario (o, al menos, así lo consideran algunos usuarios).

La integración de opciones que caracterizan la "socialización" del opac, como es el acceso a información comunitaria, tenía buenos resultados en los catálogos de bibliotecas públicas, pero no así en los del ámbito académico, que han mejorado considerablemente. En cuan- to a las facilidades prestadas para acceder a información corporativa, las mejoras también son importantes.

La implementación de los distintos tipos de ayuda ha tendido a igualarse en los dos grupos de bibliotecas estudiados, de forma que algunas de las diferencias observadas en 2005 son ahora inapreciables. Sin embargo, siguen siendo más los catálogos universitarios que refuerzan la ayuda con formatos alternativos al textual, que facilitan los procedimientos mediante el acceso a "cómo puedo...", que tienen implementada la función "encontrar" y que disponen de páginas temáticas. También es mayor la importancia que conceden las bibliotecas académicas a facilitar ayudas que expliquen la ordenación de las colecciones o cómo buscar en el opac por las materias asignadas (tabla III).

Por el contrario, a juzgar por los resultados obtenidos, el interés por desarrollar plenamente la ayuda sensible al contexto (un tipo de ayuda más clásica) sigue siendo más propio de las bibliotecas públicas. No obstante, la valoración de los resultados ha de hacerse referida al conjunto de ayudas alternativas, que cuanto mayores y diversas son, menos hacen necesaria su implementación completa. También son más las bibliotecas públicas que disponen de un servicio de FAQ's, de herramientas para facilitar la referencia en línea y de guías de ayuda personalizadas (tabla III).

Los gráficos 1 y 2 presentan la evolución en la implementación de ayudas que en 2005 lo estaban en me-

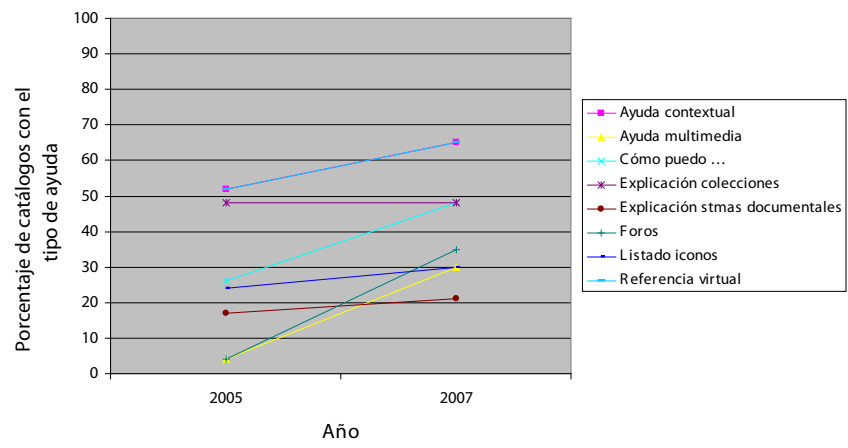

Gráfico 1. Evolución en la implementación de tipos de ayuda en OPACs de bibliotecas públicas (2005-2007)

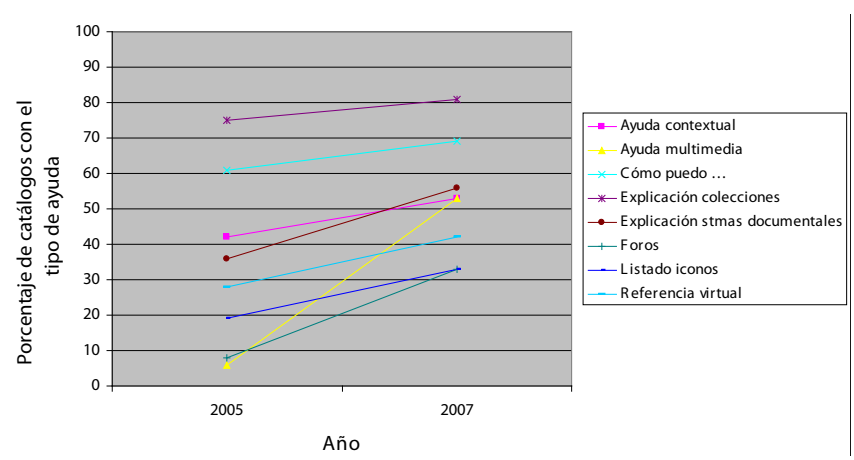

Gráfico 2. Evolución en la implementación de tipos de ayuda en OPACs de bibliotecas universitarias (2005-2007) 


\begin{tabular}{|c|c|c|c|c|}
\hline \multirow{2}{*}{ Tipos de ayuda (en \%) } & \multicolumn{2}{|c|}{ Tipos bibliotecas } & \multicolumn{2}{|c|}{ Países } \\
\hline & Universitarias & Públicas & España & EE.UU \\
\hline Ayuda sensible contexto & 53 & 65 & 64 & 56 \\
\hline Ayuda en formato multimedia & 53 & 30 & 57 & 40 \\
\hline Boletines electrónicos & 89 & 100 & 100 & 91 \\
\hline Burbuja informativa & 100 & 100 & 100 & 100 \\
\hline Cómo puedo... & 69 & 48 & 64 & 60 \\
\hline Directorio electrónico & 94 & 74 & 93 & 84 \\
\hline Ejemplos en pantallas de ayuda & 97 & 100 & 100 & 98 \\
\hline Encontrar & 92 & 87 & 93 & 89 \\
\hline Explicación de la ordenación de las colecciones & 81 & 48 & 71 & 67 \\
\hline Explicación de los sistemas documentales & 56 & 22 & 43 & 42 \\
\hline FAQ's & 69 & 78 & 64 & 76 \\
\hline Foros de participación & 33 & 35 & 21 & 38 \\
\hline Guías de ayuda personalizada & 72 & 87 & 57 & 84 \\
\hline Índice general de la ayuda & 72 & 70 & 71 & 71 \\
\hline Listado de instrucciones & 100 & 96 & 100 & 98 \\
\hline Listado explicativo de iconos & 33 & 30 & 50 & 27 \\
\hline Mapa de localización & 83 & 61 & 71 & 76 \\
\hline Páginas temáticas & 83 & 70 & 64 & 82 \\
\hline Referencia virtual & 42 & 65 & 7 & 64 \\
\hline Sistema de difusión de información e-mail & 97 & 91 & 79 & 100 \\
\hline Sugerencias y reclamaciones & 100 & 100 & 100 & 100 \\
\hline \multicolumn{5}{|c|}{ Información corporativa } \\
\hline Agenda de actividades & 92 & 100 & 100 & 93 \\
\hline Fotos, imágenes & 64 & 78 & 71 & 69 \\
\hline Encuestas de opinión & 17 & 13 & 29 & 11 \\
\hline Estadísticas & 69 & 74 & 86 & 67 \\
\hline Guía de servicios & 97 & 100 & 100 & 98 \\
\hline Guía del personal & 94 & 91 & 100 & 91 \\
\hline Guía de la organización & 97 & 100 & 100 & 98 \\
\hline Instalaciones & 83 & 83 & 93 & 80 \\
\hline Memoria & 44 & 52 & 64 & 42 \\
\hline Normas de servicios básicos & 100 & 100 & 100 & 100 \\
\hline Noticias propias & 94 & 96 & 93 & 96 \\
\hline Reglamentos & 75 & 78 & 79 & 76 \\
\hline Revistas, folletos, etc. propios & 39 & 61 & 21 & 56 \\
\hline \multicolumn{5}{|c|}{ Información comunitaria } \\
\hline Agenda cultural & 25 & 65 & 43 & 40 \\
\hline Información & 94 & 91 & 100 & 92 \\
\hline Noticias externas & 58 & 96 & 57 & 78 \\
\hline
\end{tabular}

Tabla III. Resultados comparados por tipos de bibliotecas y países

nos de la mitad de los opacs analizados, de bibliotecas públicas y universitarias.

Al comienzo de este estudio, prácticamente todas las bibliotecas habían modificado el software de ayuda estándar, pero las diferencias constatadas en el desarrollo de un mismo software en cada tipo de biblioteca avalaban la idea de que aprovechar el potencial de las herramientas informáticas depende, en buena medida, de la capacidad de las propias bibliotecas. Dos años después siguen advirtiéndose diferencias, que en algunos casos son significativas, como se observa en la tabla IV para el caso de las funciones "cómo puedo..." y "encontrar".

\section{Resultados según país}

En 2005 los opacs analizados de bibliotecas de Estados Unidos disponían de una mayor diversidad de tipos de ayuda y aprovechaban mejor el potencial de la tecnología, lo que se traducía en la implementación de ayudas más complejas de desarrollar y mantener, como las que permiten una asistencia directa en línea, por ejemplo. La mejora de los catálogos españoles en este tiempo es evidente, si bien no alcanzan el nivel de desarrollo, en este tipo de ayudas, de los norteamericanos (tabla III).

Las diferencias observadas al comienzo del estudio podían deberse a que al ser la mayoría de los softwares de origen norteamericano, allí se implementan antes las versiones más nuevas y, por tanto, con mayores posibilidades tecnológicas. Sin embargo, siendo esto cierto, los datos de 2007 hacen razonable pensar que el apoyo informático que reciben las bibliotecas españolas es menor y, también, que es insuficiente el personal con 


\begin{tabular}{|c|c|c|c|c|c|c|c|c|c|c|}
\hline \multirow{2}{*}{$\begin{array}{l}\text { OPCIÓN } \\
\text { (en \%) }\end{array}$} & \multicolumn{2}{|c|}{$\begin{array}{l}\text { CARL/LIBRARY } \\
\text { SOLUTION } ®\end{array}$} & \multicolumn{2}{|c|}{ SIRSI-HORIZON } & \multicolumn{2}{|c|}{$\begin{array}{l}\text { INNOPAC- } \\
\text { MILLENIUM }\end{array}$} & \multicolumn{2}{|c|}{ SIRSI-UNICORN $®$} & \multicolumn{2}{|c|}{ BARATZ-ABSYS } \\
\hline & $\begin{array}{l}\text { Univer- } \\
\text { sidades }\end{array}$ & $\begin{array}{c}\text { B. } \\
\text { Públicas }\end{array}$ & $\begin{array}{l}\text { Univer- } \\
\text { sidades }\end{array}$ & $\begin{array}{c}\text { B. } \\
\text { Públicas }\end{array}$ & $\begin{array}{l}\text { Univer- } \\
\text { sidades }\end{array}$ & $\begin{array}{l}\text { B. } \\
\text { Públicas }\end{array}$ & $\begin{array}{l}\text { Univer- } \\
\text { sidades }\end{array}$ & $\begin{array}{c}\text { B. } \\
\text { Públicas }\end{array}$ & $\begin{array}{l}\text { Univer- } \\
\text { sidades }\end{array}$ & $\begin{array}{l}\text { B. } \\
\text { Públicas }\end{array}$ \\
\hline Cómo puedo... & 20 & 60 & 60 & 60 & 78 & 17 & 89 & 80 & 100 & 0 \\
\hline Encontrar & 80 & 80 & 80 & 60 & 89 & 67 & 100 & 100 & 100 & 100 \\
\hline
\end{tabular}

Tabla IV. Ejemplos de opciones implementadas según software y tipo debiblioteca

\section{"El apoyo informático a las bibliotecas es clave para aprovechar al máximo el potencial del software del opac y del entorno web, como también lo es un perfil del personal bibliotecario que reúna habilidades y competencias informáticas"}

un perfil que reúna las necesarias competencias y habilidades informáticas. Porque, como ya se ha comentado, explotar al máximo las posibilidades del software está en manos de la biblioteca.

No obstante, los resultados referidos a los opacs españoles denotan un claro progreso en la implementación de una gran parte de las opciones de ayuda que en 2005 no estaban disponibles en la mitad de los evaluados (gráfico 4), evolución que no se corresponde con el escaso desarrollo de herramientas que facilitan una referencia virtual o permiten a los usuarios participar en foros.

Si se comparan los gráficos 3 y 4, que presentan la evolución en la implementación de ayudas que en 2005 no lo estaban en la mitad de los catálogos españoles o norteamericanos, se observa que el incremento en el porcentaje de opacs se acentúa considerablemente en el caso de los españoles, mostrando una clara tendencia a igualarse con los de Estados Unidos.

Es significativo el avance experimentado en las funciones "cómo puedo..." y "encontrar", y también en la implementación de FAQs. Respecto a las FAQs y "cómo puedo..." es preciso señalar que mientras en los norteamericanos existe una clara tendencia a individualizarlas en dos tipos de ayuda diferenciadas, en los españoles, en algunos casos, la función "cómo puedo..." se confunde con el servicio de FAQs e, incluso, aparecen mezclados.

Las pantallas de las bibliotecas de la Universidad de Granada y de la Universidad de Washington son dos ejemplos de implementación de la función "cómo puedo...". En la del opac de la Universidad de Washington se ve también el acceso a un servicio de referencia mediante chat, así como a información sobre la biblioteca. Por otra parte, la pantalla del catálogo de la Universidad de Arizona muestra un ejemplo de ayuda interactiva.

A la personalización de guías de ayuda siguen concediendo mayor importancia las bibliotecas de Estados Unidos, mientras que el acceso a información corporativa es más completo en las españolas. Y si hace dos años los usuarios de catálogos norteamericanos tenían más facilidades para acceder a información comunitaria, en la actualidad las diferencias son inexistentes.

Por otra parte, son más los opacs de bibliotecas españolas que tienen implementada una ayuda contextual completa, los que facilitan una explicación de los iconos y de la ubicación de los ejemplares, así como los que utilizan formatos alternativos al textual.

\section{Conclusiones}

El entorno hipertextual ha solucionado el problema de la necesaria ubicuidad de la información, favore-

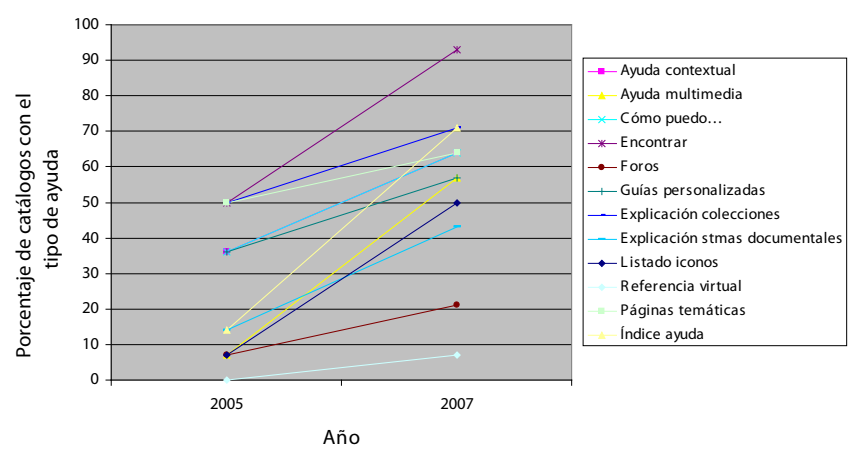

Gráfico 3. Evolución en la implementación de tipos de ayuda en OPACs de bibliotecas españolas

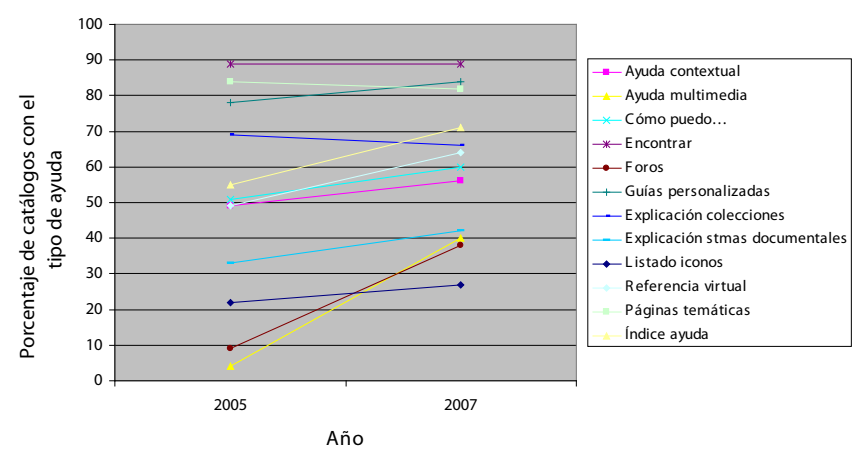

Gráfico 4. Evolución en la implementación de tipos de ayuda en OPACs de bibliotecas norteamericanas 


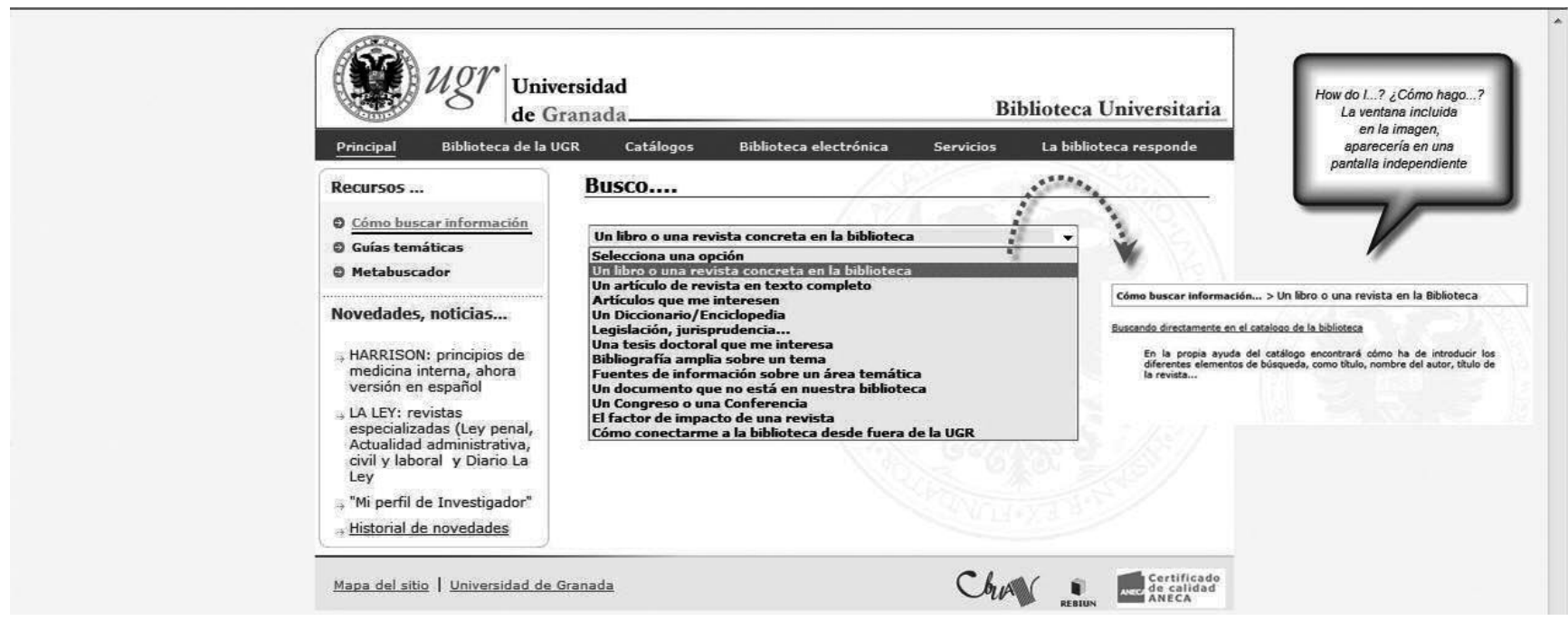

Ejemplo 3. Pantalla de ayuda de la biblioteca de la Universidad de Granada que explica cómo buscar información según los distintos tipos de documentos

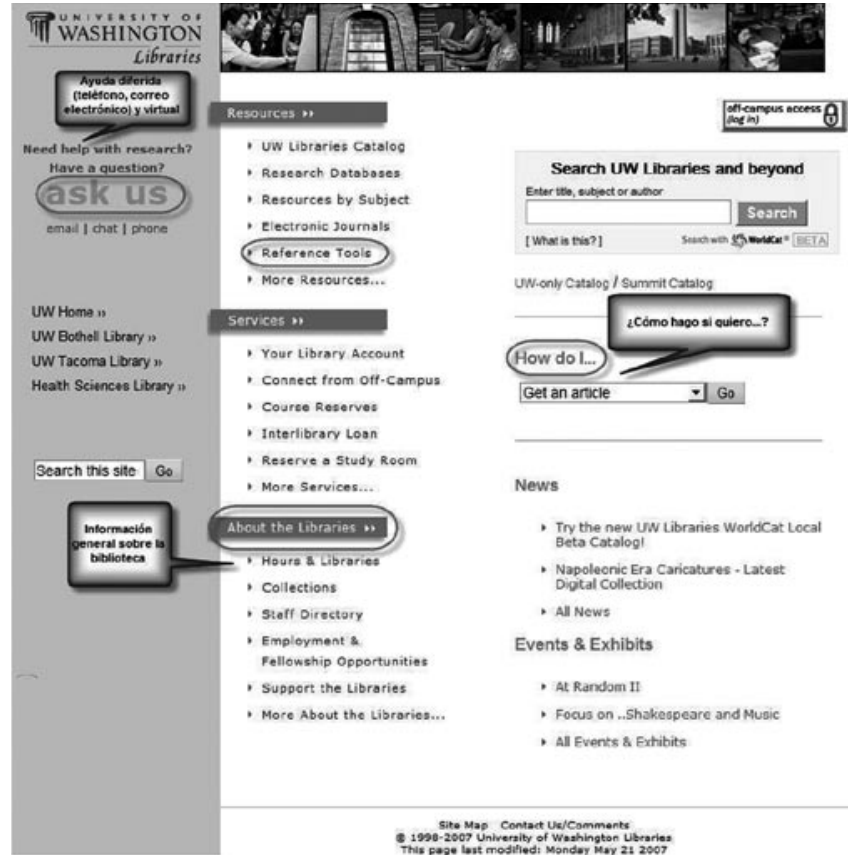

Ejemplo 4. Pantalla de ayuda del catálogo de la Universidad de Washington que muestra el acceso a diferentes tipos de ayuda

ciendo el desarrollo de una idea de ayuda más amplia y haciendo de los opacs herramientas más potentes en la asistencia que prestan a sus usuarios. Asimismo, el auge de servicios disponibles en la Red, con sofisticadas herramientas de apoyo, ha influido en el alcance y función del propio catálogo y su sistema de ayuda. Y aunque todavía hoy se advierten diferencias entre estos otros servicios y los del ámbito bibliotecario, la distancia se ha acortado considerablemente.

Según los resultados de este estudio, el ritmo de adecuación del opac a las posibilidades del desarrollo tecnológico es menor que el que se observa en otros productos informáticos. Las bibliotecas tardan más en explotar el potencial de las tecnologías, quizás porque no se desarrolla pensando en ellas, lo que las obliga a adaptar lo creado para otros ámbitos. Quizás, también, porque gozan de menos apoyo informático del necesario, o porque el perfil de su personal no se adecua plenamente a las exigencias del mercado de la informática. Consideraciones, estas últimas, que se acentúan en el caso de los catálogos de bibliotecas españolas.

Ahora bien, los actuales opacs disponen de sistemas de ayuda más flexibles y adaptables a las singularidades y nivel de dominio de los usuarios, e incorporan una mayor diversidad de tipos y puntos de acceso a la ayuda, algunos de ellos de compleja implementación y mantenimiento. Integran, además, herramientas y prestaciones complementarias a las ayudas tradicionales, que ahora forman parte de la estructura inherente del opac.

Todo ello proyecta un panorama muy favorable, especialmente visible en los catálogos de bibliotecas españolas, cuya evolución en estos dos últimos años los sitúa en un nivel de desarrollo similar al de los de bibliotecas norteamericanas, excepto en lo que se refiere a la implementación de herramientas de participación y comunicación. Queda todavía pendiente, en una gran mayoría, facilitar a los usuarios verdaderos servicios de referencia virtual, así como la posibilidad de participar en foros.

El concepto de opac híbrido se hace visible, una vez se comprueba que opac y portal comparten ayudas y puntos de acceso. Las respuestas a las preguntas más frecuentes cobran cada vez más importancia, y también las opciones que permiten comunicaciones instantáneas y directas, entre usuarios y entre éstos y bibliotecarios, que comienzan a ganar terreno sobre otros tipos de ayuda más clásicos, como el correo electrónico.

El uso de formatos multimedia o con algún tipo de animación para facilitar la comprensión de un proceso es un recurso todavía poco utilizado, si bien se percibe un progreso importante, especialmente en los catálogos de bibliotecas españolas. 


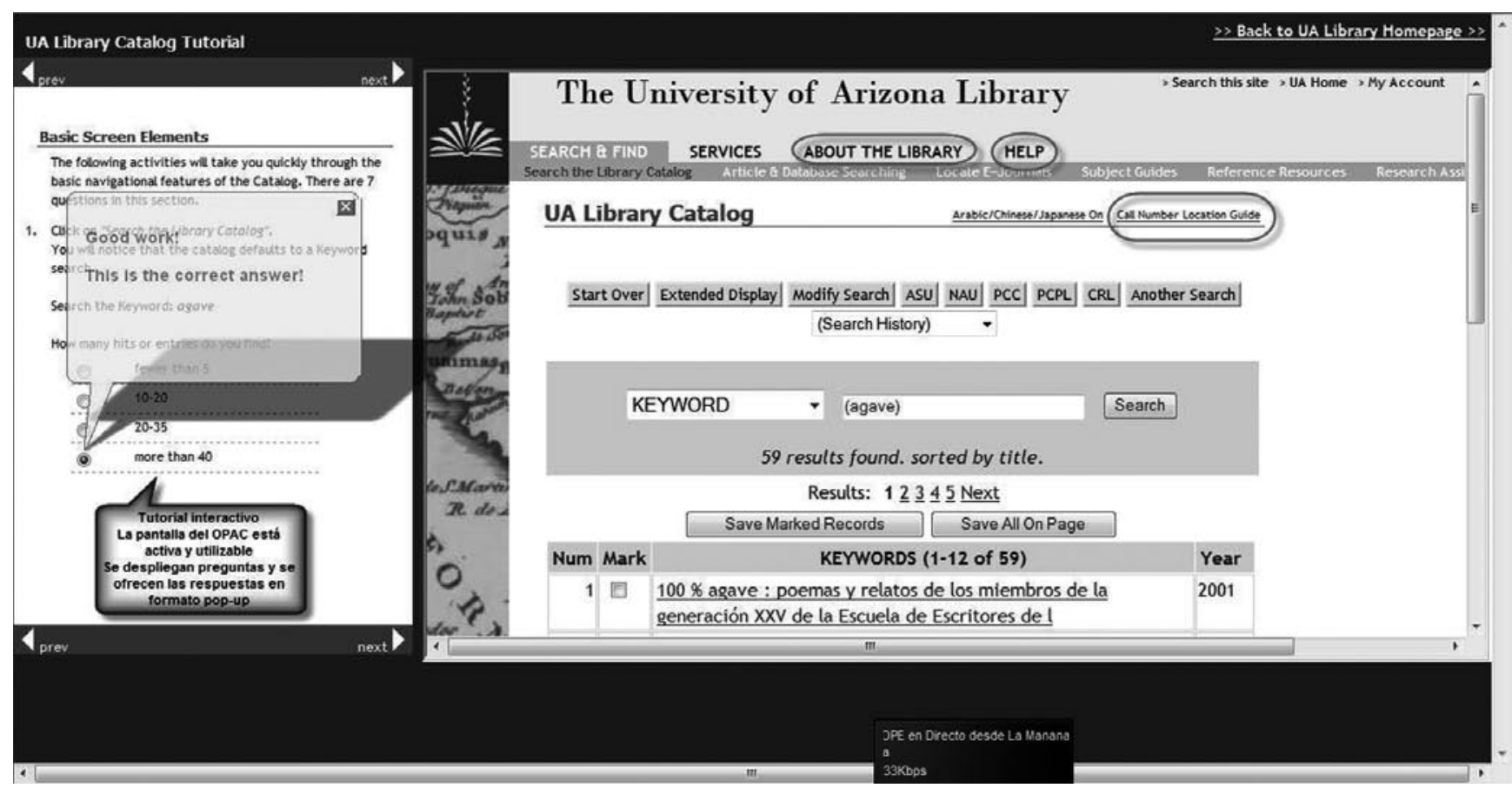

Ejemplo 5. Pantalla de ayuda del catálogo de la Universidad de Arizona que muestra un tutorial interactivo así como el acceso a otros tipos de ayuda

\section{Notas}

1. Combina las características del estudio intencional (caracterizado por un esfuerzo deliberado de obtención de muestras representativas mediante la incorporación de grupos supuestamente típicos), con las del estudio por cuotas, en el que se establecen unos cupos suficientemente representativos para que la muestra se considere válida, basándose en un buen conocimiento de los estratos de la población (individuos más representativos o adecuados para los fines de la investigación).

2. WritersUA es una compañía especializada en la prestación de programas de formación y publicaciones relacionados con la asistencia al usuario. http://www.writersua.com

Esta dirección es un punto de encuentro para los profesionales que trabajan en la creación de estos sistemas.

3. Se incluye como ejemplo la ayuda insertada en la pantalla de búsqueda del opac de la Universidad de Oregón, que explica cómo buscar información a partir de los encabezamientos de la Biblioteca del Congreso (LCSH).

4. Se incluye como ejemplo de referencia virtual una pantalla de la biblioteca de la Universidad de Murcia.

5. Las encuestas de opinión se han analizado en los casos que estaban activas en el momento de la evaluación.

6. Los porcentajes de todas las tablas y gráficos se han redondeado.

7. Los resultados del estudio The 2001 WinWriters skills and technologies survey concluyen que el índice general es una de las ayudas más utilizadas por los creadores del software (el $90 \%$ lo usaron en sus desarrollos de ayuda). Joe Welinske. "2001 Skills and technologies survey". En: WinWriters: training for software user assistance.

http://members.chello.nl/c.huijbers1/texpert1/skillstech01.pdf http://www.winwriters.com/skillstech01.htm

\section{Referencias}

Adams, Wendy. "The effectiveness of online help systems: text only, animated images only, and integrated interactive" [en línea]. En: SHORE 2001: Student HCI Online Research Experiments, 2001. College Park: University of Maryland, 2001 [Consulta: 12 enero 2007].

http://www.otal.umd.edu/SHORE2001/help/index.html

Ebenezer, Catherine. "Trends in integrated library systems" [en línea]. En: VINE, 2003, v. 34, n. 4, pp. 19-45. [Consulta: 22 septiembre 2006].
http://members.lycos.co.uk/ebenezer1954/Whither_integrated_library_systems.doc

Ellison, Matthew. "A usability test of web-based user assistance" [en línea]. En: WinWriters: training for software user assistance. Seattle: WritersUA, 2002 [Consulta: 28 enero 2007].

http://www.writersua.com/usability_test_analysis.htm

Evans, Sarah E. "The catalog as portal to the Internet" [en línea]. En: Proceedings of the Bicentennial conference on bibliographic control for the new millennium: confronting the challenges of networked resources and the web, Washington, D.C., Library of Congress, Cataloging Distribution Service, 2001, pp. 21-37. [Consulta 10 septiembre 2006].

http://www.loc.gov/catdir/bibcontrol/thomas_paper.html

Kearsley, Greg. Online help systems: design and implementation. Noorwood, NJ: Ablex, 1988. ISBN. 089391472X

Nielsen, Jakob. Jakob Nielsen's alertbox : current issues in web usability: June 24, 2001 [Consulta: 16 agosto 2006].

http://www.useit.com/alertbox/20010624.html

Shneiderman, Ben. Designing the user interface: strategies for effective human-computer interaction. $3^{\text {rd }}$ ed. Reading, MA: Addison-Wesley, 1998, ISBN 0201694972.

Weber, Jean Hollis. Is the help helpful? How to create online help that meets your users' needs. Whitefish Bay, WI: Hentzenwerke Publishing, 2004, ISBN 1930919603.

White, Frank. "An evaluation of online help for the NOTIS OPAC". En: Library software review. 1994, Spring, v. 13, n. 1, pp. 4-17.

Purificación Moscoso, Universidad de Alcalá, Plaza de San Diego s/n, 28801 Alcalá de Henares, Madrid. p.moscoso@uah.es

Francisco-Manuel García-Ortiz, 1036 Prosser Ave., Prosser, WA 99350

Tel.: +1-5097812268

invesopac@yahoo.com 
